\title{
Correction to: Tolerability and toxicity of trastuzumab or trastuzumab + lapatinib in older patients: a sub-analysis of the ALTTO trial (BIG 2-06; NCCTG (Alliance) N063D)
}

\author{
Noam Pondé ${ }^{1,2}$ (D) Dominique Agbor-Tarh ${ }^{3} \cdot$ Lissandra Dal Lago $^{2} \cdot$ Larissa A. Korde $^{4} \cdot$ Florentine Hilbers $^{5}$. \\ Christian Jackisch $^{6}$. Olena Werner ${ }^{7} \cdot$ Richard D. Gelber $^{8,9} \cdot$ Aminah Jatoi $^{10} \cdot$ Amylou C. Dueck $^{11}$. \\ Alvaro Moreno-Aspitia ${ }^{12} \cdot$ Christos Sotiriou ${ }^{2} \cdot$ Evandro de Azambuja $^{2} \cdot$ Martine Piccart $^{13}$
}

Published online: 8 October 2021

(c) Springer Science+Business Media, LLC, part of Springer Nature 2021

\section{Correction to: \\ Breast Cancer Research and Treatment (2021) 185:107-116 https://doi.org/10.1007/s10549-020-05915-9}

In the original publication of the article, the funding information was published incorrectly. Now the same has been corrected and published here.
The ALTTO Trial, as well as this subanalysis, has been funded NIH grant (U10 CA180821), GSK and Novartis.

Publisher's Note Springer Nature remains neutral with regard to jurisdictional claims in published maps and institutional affiliations.
The original article can be found online at https://doi.org/10.1007/ s10549-020-05915-9.

Noam Pondé

noam.ponde@accamargo.org.br

1 AC Camargo Cancer Center, Rua Pires da Mota, São Paulo 1167, Brazil

2 Institut Jules Bordet, Brussels, Belgium

3 Frontier Science Scotland (FSS), Kincraig, UK

4 National Cancer Institute, Bethesda, MD, USA

5 Breast International Group, Brussels, Belgium

6 Sana Klinikum, Offenbach, Germany

\section{Novartis, Basel-City, Switzerland}

Dana-Farber Cancer Institute, Boston, USA

9 Frontier Science and Technology Research Foundation, Boston, MA, USA

10 Mayo Clinic, Rochester, MN, USA

11 Mayo Clinic, Scottsdale, AZ, USA

12 Mayo Clinic, Jacksonville, FL, USA

13 Institut Jules Bordet, Université Libre de Bruxelles (ULB), Brussels, Belgium 